\title{
Perfil epidemiológico de los pacientes con cuerpos extraños en el Servicio de Otorrinolaringología del Hospital Central de Maputo observados de 1983 a 2009
}

\author{
Epidemiological profile of patients with strange bodies in the \\ Otorhinolaryngology Service of the Central Hospital \\ of Maputo observed from 1983 to 2009
}

\author{
Mahomed S. Dadá1,2, Mohsin Sidat ${ }^{3}$.
}

\begin{abstract}
RESUMEN
Introducción: En los países en desarrollo la problemática de cuerpos extraños (CE) en los Servicios de Otorrinolaringología está mal estudiada, la literatura sobre el tema es escasa. El estudio fue realizado para caracterizar el perfil epidemiológico en pacientes atendidos con CE en el Servicio de ORL/Hospital Central de Maputo (HCM) (período de 1983 a 2009).

Objetivo: El estudio fue realizado para caracterizar el perfil epidemiológico en pacientes atendidos con cuerpos extraños en el Servicio de ORL del Hospital Central de Maputo (HCM) en el período de 1983 a 2009.

Material y Método: Se realizó un estudio transversal utilizando datos retrospectivos de pacientes con cuerpos extraños atendidos en el HCM. Se recogieron datos demográficos, sobre CE, ubicación anatómica, tratamientos efectuados y complicaciones clínicas. Los datos se procesaron utilizando el SPSS-v15. Los resultados descriptivos se muestran en tablas y gráficos.

Resultados: Se incluyeron un total de 4.826 pacientes. Se observó predominio del sexo masculino y la mayoría pertenecía a la edad pediátrica. Los CE predominantes fueron los metales y la espina de pescado. La región anatómica más afectada fue el canal auditivo externo.

Conclusión: Este estudio presenta una compilación única que abarca un período de 26 años constituyendo una importante fuente de información al respecto. Demuestra la magnitud del problema en la edad pediátrica y presenta las características de los CE involucrados y las respectivas localizaciones anatómicas.
\end{abstract}

Palabras clave: Cuerpos extraños, otorrinolaringología, Mozambique.

\footnotetext{
1 Servicio de Otorrinolaringología. Hospital Central de Maputo. Mozambique.

2 Departamento de Ciencias Morfológicas, Facultad de Medicina. Universidad Eduardo Mondlane. Maputo. Mozambique.

3 Departamento de Salud de la Comunidad, Facultad de Medicina. Universidad Eduardo Mondlane. Maputo. Mozambique.

Recibido el 20 de septiembre, 2017. Aceptado el 5 de noviembre, 2017.
} 


\section{ABSTRACT}

Introduction: In developing countries, the problem of foreign bodies (FB) in ENT Services is poorly studied and the literature related to the subject is rare. This study was performed to characterize the epidemiological profile in patients treated with FB in ENT Department/Maputo Central Hospital (MCH) (1983-2009 period).

Aim: This study was performed to characterize the epidemiological profile in patients treated with foreign bodies in ENT Department of Maputo Central Hospital (MCH) in the 1983-2009 period.

Material and Method: A cross-sectional study was performed using the retrospective data from patients with $F B$ treated at $M C H$. Demographic data were collected on $F B$, their anatomical location, any treatment, clinical complications among others. The data were processed using the SPSS-v15. Descriptive results were presented in tables and graphs.

Results: We included a total of 4826 patients. The most affected population groups were males and children under 14 years. The most frequent $F B$ were metals. The most common anatomical locations of FB was the external ear canal.

Conclusions: This study presents a unique collection covering a period of 26 years, constituting, therefore, an important source of information on the subject. This study demonstrates the magnitude of the problem in the pediatric age and presents the characteristics of the FB involved and their anatomical locations. The evidence of this study are important to inform clinical prevention and training strategies to deal with this issue at the level of primary health care. This study is important to inform about strategies to prevent it, but also useful in the formation of clinics to better deal with this type of patients at the level of primary health care.

Key words: Foreign bodies, otorhinolaryngology, Mozambique.

\section{INTRODUCCIÓN}

En países en desarrollo la problemática de cuerpos extraños (CE) en los Servicios de Otorrinolaringología (ORL) está mal estudiada. La literatura no refleja la magnitud de la problemática, sin embargo estudios publicados muestran que Ios CE en los servicios de ORL representan un importante problema de salud'. LOS CE producen trastornos físicos, emocionales y económicos en las personas dañadas y en los Servicios de Salud.

La literatura focaliza el tema de Ios CE como un problema clínico, existiendo pocos estudios que abarquen el impacto en la salud pública'. Según la literatura, los niños son los más afectados². Varios autores señalan la penetración de un CE en orificios naturales de cabeza y cuello (ONCC), su ingesta y/o inhalación como una de las principales causas de accidentes domésticos en niños ${ }^{3}$. Una complicación común es la obstrucción aérea4. Las complicaciones no fatales dejan secuelas crónicas que afectan la calidad de vida ${ }^{5}$.
En adultos, la causalidad y tipología son diferentes. Se producen por colocación de objetos en la boca, el enclavamiento del bolo alimentario de gran tamaño o por una enfermedad que impida la progresión de alimentos por el esófago ${ }^{6}$.

Los CE en las Urgencias de ORL varían de $4 \%^{3}$ a $41,3 \% 7$, dependiendo de la unidad sanitaria y de la población de estudio. En estudios publicados del tipo Audit, el peso de CE en ORL varía de 3,5\% en Grecia $^{8}$ a $44,2 \%$ en la India ${ }^{9}$. Así, este trabajo pretende reportar el perfil epidemiológico de pacientes con CE observado en el Servicio de ORL/HCM, en el período de 1983-2009.

\section{MATERIAL Y MÉTODO}

Se trata de un estudio observacional/transversal/ descriptivo que recurrió a la recogida y análisis de los datos secundarios disponibles en los libros de registros de las Consultas/Urgencias/Salas de tratamiento de ORL y de los Bloque Central/ 
ORL/SUR del HCM y de las fichas clínicas de los pacientes. Se incluyeron datos de todos los pacientes con CE de forma consecutiva en el HCM $(1 / 1 / 1983-31 / 12 / 2009)$.

Los pacientes con más de un CE en la misma área anatómica fueron considerados como un solo individuo. Los individuos de 0-14 años se consideraron pacientes pediátricos de acuerdo con la legislación de Mozambique ${ }^{10}$. Se garantizó la confidencialidad de los datos a través del anonimato, el uso de contraseñas y del acceso a los datos sólo a los investigadores.

Se recogieron los siguientes datos: fecha, día de la semana, edad, sexo, naturaleza, tipo de CE, localización anatómica, procedimiento, uso o no de anestesia y complicaciones derivadas del CE y/o de los procedimientos. Se definieron como criterios de inclusión: presencia confirmada del CE en los ONCC. Los criterios de exclusión fueron: presencia no confirmada del CE y aquellos cuya información clínica era incompleta.

Los datos se introdujeron en una base de datos en el paquete estadístico SPSS-v15. Este estudio tuvo las debidas aprobaciones éticas.

\section{RESULTADOS}

En el período de estudio se analizaron datos de 5.483 pacientes con CE en los ONCC, excluyendo 657 por datos incompletos, quedando 4.826 .
La mayoría de los pacientes observados fue de género masculino $(52,8 \%, 2.549 / 4.826)$. La edad media de los pacientes fue de 14,7 años (mediana de 7,0 y desviación estándar de 15,7 años, el rango de variación fue de 2 meses a los 82 años), con predominancia de pacientes con edad menor 0 igual a 14 años $(64,9 \%, 3.130 / 4.826)$. La mayoría procedían de Maputo $(87,5 \%, 4.222 / 4.826)$, de Gaza $(4,60 \%, 222 / 4.826)$ y de Inhambane $(3,20 \%$, 156/4.826). La Figura 1 muestra los casos ocurridos de acuerdo a los meses del año. Los picos de casos se registraron en los meses de marzo y agosto. No existe diferencia significativa en la aparición de casos con CE durante los diferentes días hábiles de la semana, pero en los fines de semana se observó un menor número de casos (Figura 2).

Los CE pueden ser clasificados como orgánicos e inorgánicos ${ }^{11}$. Los orgánicos pueden ser del tipo alimenticio (espinas de pescado/huesos de animales, semillas/granos) e insectos vivos 0 muertos, mientras que los inorgánicos pueden ser compuestos de metales, plásticos, vidrio, esponja, papel, caucho. Los grupos de edad más afectados fueron de 0-14 años, representando al grupo pediátrico en el contexto de atención en Mozambique $^{10}$. Los inorgánicos fueron predominantes en esta población pediátrica. En los individuos con 15 0 más años de edad, los orgánicos fueron los de mayor ocurrencia (Figura 3).

En ambos sexos, los metales fueron más frecuente, responsable del $24,5 \%(1.181 / 4.826)$ de

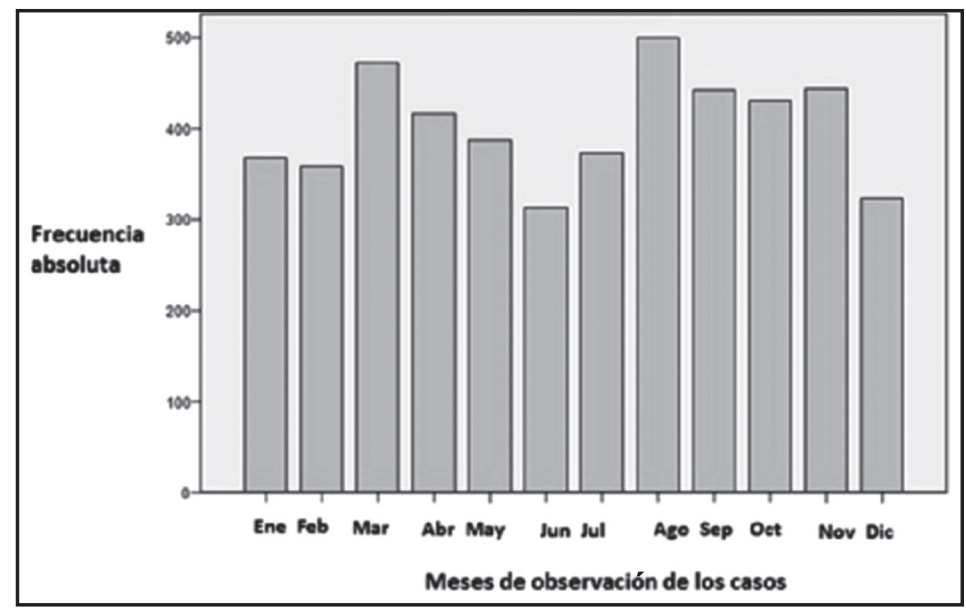

Figura 1. Distribución por meses del año de los pacientes con CE observados en el Servicio de ORL/HCM (período 1983-2009). 


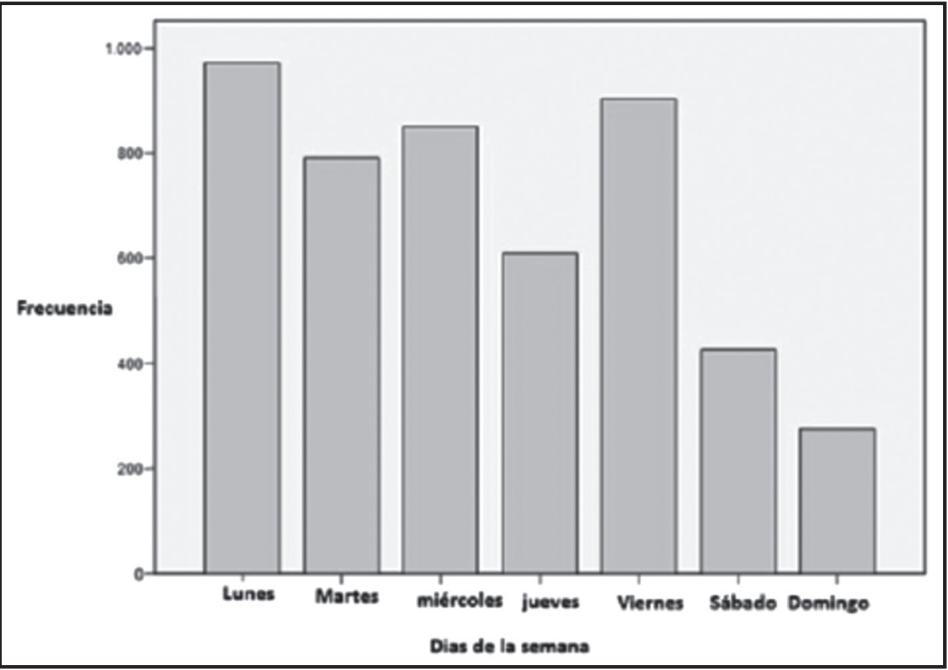

Figura 2. Distribución por días de semana de los pacientes con CE observados en el Servicio de ORL/HCM (período 1983-2009).

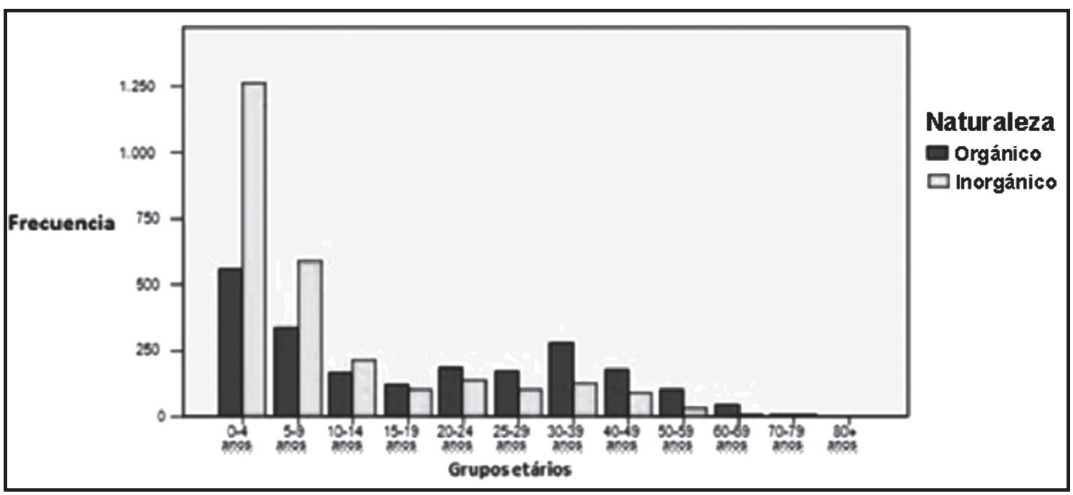

Figura 3. Distribución del tipo de CE en pacientes de los diferentes grupos de edad observados en el Servicio de ORL/HCM (período 1983-2009).

todos los CE diagnosticados, seguido de espina de pescado $(21,9 \%, 1.058 / 4.826)$ y semillas/granos $(18,9 \%, 911 / 4.826)$. Si analizamos los diferentes tipos de CE por sexo encontramos que los metales, con $27 \%$ (688/2.549), seguidos por las semillas $(20,2 \%, 514 / 2.549)$ constituyeron la mayor frecuencia en el sexo masculino mientras que la espina de pescado/hueso $(24,4 \%, 555 / 2.277)$, seguidos por los metales $(21,65 \%, 493 / 2.277)$ constituyeron la mayor frecuencia en el sexo femenino (Figura 4).

La Tabla 1 muestra la distribución de los CE según regiones anatómicas. El CAE fue la región anatómica con mayor presencia de CE $(40,3 \%)$, seguido del esófago (31,90\%).

Los CE de origen metálico se encontraron predominantemente en el esófago (85,80\%, $1.014 / 1.181)$, la mayoría consistían en monedas $(79,80 \%, 943 / 1.181)$. Los objetos de madera eran más frecuentes en el canal auditivo externo (CAE) $(92,38 \%, 97 / 105)$. Los fragmentos de vidrio se encontraron mayoritariamente en el esófago $(56,25 \%, 18 / 32)$. El algodón en el 97,5\% (475/487) de los casos en el CAE. Los CE de origen vegetal fueron frecuentes en el CAE $(71,7 \%, 653 / 911)$ y las fosas nasales $(21,18 \%, 193 / 911)$. 


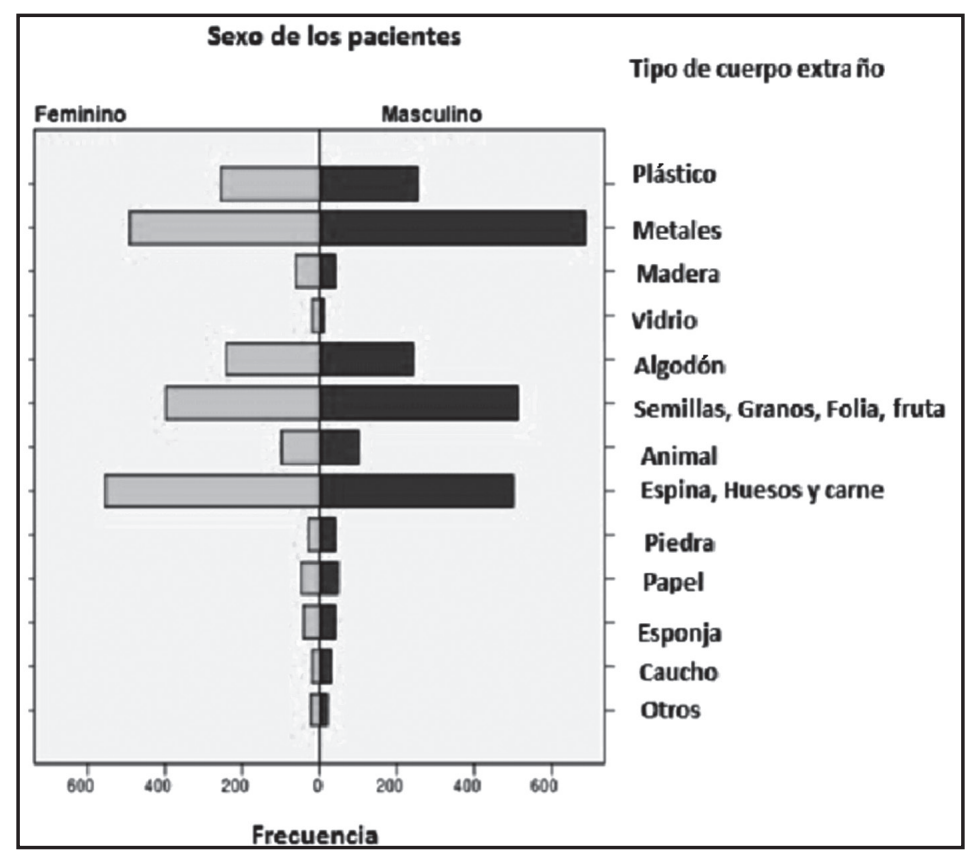

Figura 4. Distribución de los diferentes tipos de CE en pacientes de acuerdo con el sexo en el Servicio de ORL/HCM (período de 1983-2009).

Las espinas, los huesos y los trozos de carne se observaron principalmente en la faringe $(51,6 \%, 546 / 1.058)$ y esófago $(43 \%, 455 / 1.058)$. Las espinas fueron las más frecuentes $(85,50 \%$, 905/1.058). Los CE animales fueron encontrados frecuentemente en el CAE $(99,49 \%, 198 / 199)$. Las piedras se observaron sobre todo en el CAE $(71 \%$, $49 / 69)$ y en las fosas nasales $(26 \%, 18 / 69)$.

El papel prevaleció en el CAE $(63,2 \%, 62 / 98)$ y en las fosas nasales $(36,7 \%, 36 / 98)$. La esponja fue encontrada frecuentemente en las fosas nasales $(84,5 \%, 71 / 84)$. La goma de borrar de lápices se observó predominantemente en el CAE $(66 \%$, $33 / 50$ y en las fosas nasales $(22 \%, 11 / 50)$.

En la Tabla 1 podemos observar que en las vías aéreas las semillas/granos son los más frecuentes $(27,95 \%, 45 / 161)$ seguidas de espina $(22,36 \%$, $36 / 161)$ y plásticos $(21,73 \%, 35 / 161)$. En la vía digestiva, los CE más frecuentes fueron los metales $(48,16 \%, 1.023 / 2.124)$, espina/huesos $(47,45 \%$, $1.008 / 2.124)$ y plásticos $(2,10 \%, 46 / 2.124)$.

La mayoría de los pacientes observados con CE durante el periodo del estudio tenía menos de
14 años. En el grupo de 0-4 años de edad fueron observados 1.823 casos $(37,8 \%)$ y en grupo dos 5-9 años fueron observados 926 casos (19,2\%). En los niños de 0-4 años hubo predominio del CE esofágicos $(34,5 \%, 629 / 1.823)$, seguido de auriculares $(28,46 \%, 519 / 1.823)$ y nasales $(27,9 \%$, $509 / 1.823)$. En los niños de 5-9 años hubo predominio de CE auriculares $(57,12 \%, 529 / 926)$ y $30,3 \%$ (281/926) en el esófago y solamente $7,20 \%$ (67/926) en fosas nasales.

Más de la mitad de CE laríngeos (62,79\%, 27/43) aparecen en grupo pediátrico de los 0-14 años, siendo $51 \%(22 / 43)$ en el grupo etario de 0-4 años, mientras que los CE faríngeos son más frecuentes en adultos $(80,5 \%)$. Los CE traqueales son más frecuentes en grupo pediátrico de 0-14 años $(85,7 \%)$, con $66,6 \%$ en grupo de $0-4$ años, $14,28 \%$ en grupos $5-9$ años. Se realizó una traqueotomía en una niña de 18 años que aspiró una semilla. También hubo dos pacientes adultos con traqueotomías por otras causas.

Los CE laríngeos fueron extraídos usando el laringoscopio rígido en $67,40 \%(29 / 43)$ de los 


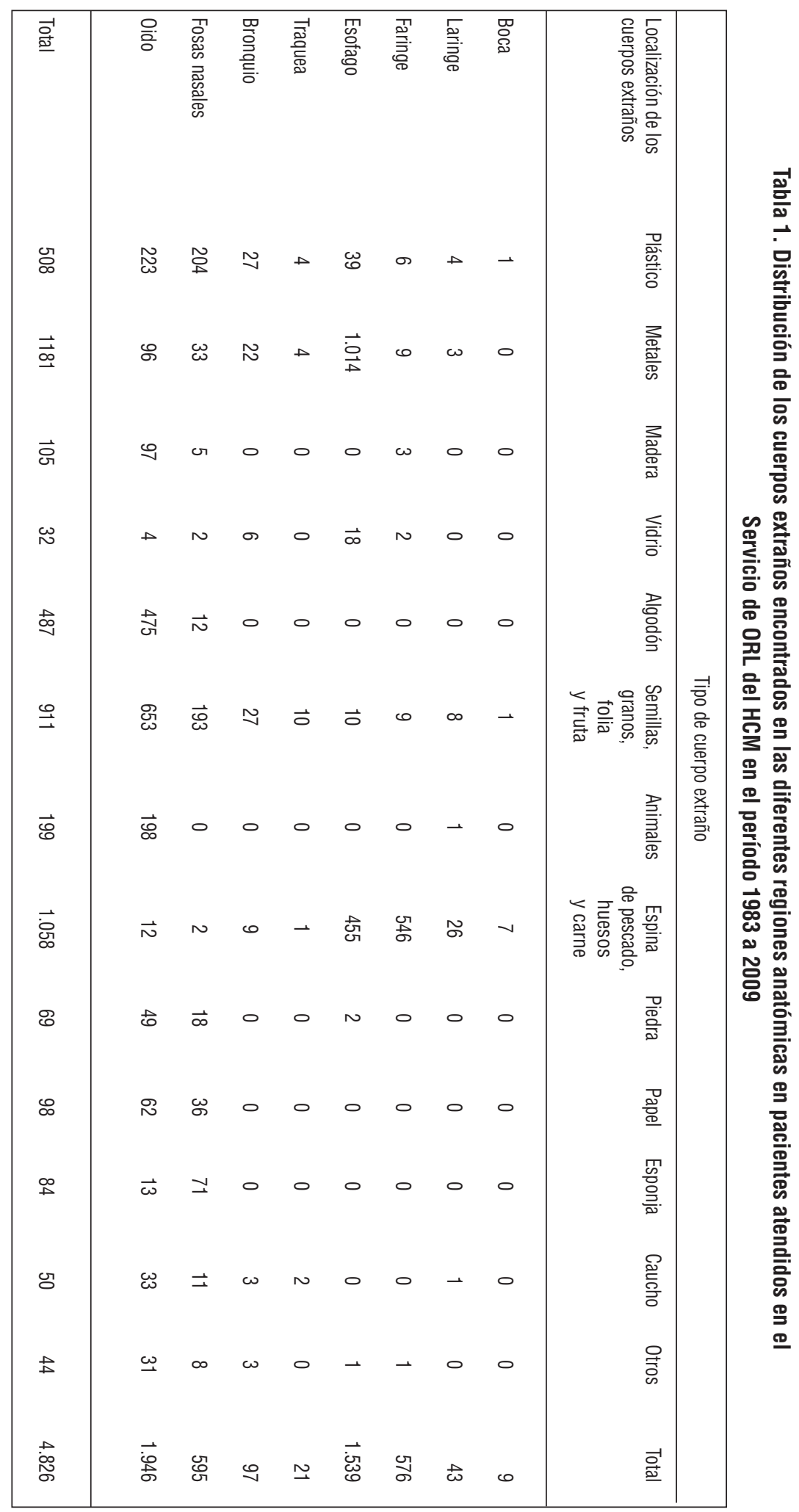


casos y en $30,20 \%(13 / 43)$ de los casos se usó laringoscopia de McTosh. Un CE fue extraído con broncoscopio rígido. No se pudo saber cuál es el bronquio más frecuentemente afectado.

La mayoría de los CE esofágicos fueron extraídos con el esofagoscopio rígido $(96,1 \%$, $1.479 / 1.539$ ) de los casos, mientras que 0,50\% se usó laringoscopio de McTosh. La expulsión espontánea ocurrió en $3,24 \%$. En dos casos hubo necesidad de hacer esofagectomía.

Todas esofagoscopías y broncoscopías rígidas fueron hechas con anestesia general. Hubo un óbito por parada cardiorrespiratoria y hemorragia bronquial posbroncoscopía con extracción de CE bronquial. Hubo una muerte por perforación del esófago y mediastinitis por CE esofágico.

\section{DISCUSIÓN}

El presente estudio presenta una análisis de los datos de pacientes con CE atendidos en el Servicio de ORL/HCM en el período de 1983-2009. El estudio presenta resultados importantes teniendo en cuenta la escasez de evidencias sobre el tema en el país y a nivel internacional. Sin embargo, la extrapolación o generalización de los resultados debe ser hecha de forma cautelosa pues el estudio fue hecho con base en los datos secundarios obtenidos sólo de una unidad hospitalaria de referencia en el país y sobre todo a nivel de la ciudad/provincia de Maputo. Cabe mencionar las limitaciones en lo que concierne a la generalización de los resultados a nivel poblacional, pues no se conoce a la población expuesta al riesgo de tener CE en IOS ONCC. Además, como no se puede asegurar que todos los pacientes que concurrieron al HCM con quejas de CE fueron referidos para el Servicio de ORL y/o si sus datos fueron adecuadamente anotados para reflejar la real situación de esa nosología en esa unidad hospitalaria.

Los hábitos, costumbres y otros factores contextuales locales contribuyen a la ocurrencia y magnitud del problema de los CE y, por lo tanto, la generalización de los resultados de este estudio se ve limitada a otros contextos diferentes. Finalmente, hay que considerar las limitaciones propias de los estudios basados en datos retrospectivos, como la falta de información verificada para el tipo de complicación no permitiendo conclusiones más precisas. Sin embargo, este estudio presenta una recopilación única de casos de pacientes con CE en Ios ONCC que abarca un período largo, constituyendo una importante fuente de información sobre el tema.

En un estudio publicado en el año 2004 sobre las urgencias de ORL/HCM, el peso del CE fue de alrededor del $25 \%{ }^{12}$. Sudáfrica reportó el nivel más bajo de ocurrencia de CE en ORL con $4 \%{ }^{3}$, y el más alto lo reportó Ghana con $41 \% 7$. Sin embargo, hay que señalar que múltiples factores socioculturales y otros pueden influir en la existencia de $C E^{13} \mathrm{y}$, como consecuencia, determinar el peso o la magnitud del problema en los servicios de urgencias ${ }^{13}$. Creemos que la cantidad de CE encontrado en este estudio es relativamente alto. En futuros estudios epidemiológicos hospitalarios o poblacionales hay que determinar, la magnitud del problema. Por otro lado, los datos disponibles y analizados en el estudio no permitieron medir sobre la relación de los CE con Ios factores socioculturales y otros. Los resultados del estudio no permiten concluir sobre la existencia de estacionalidad, ya que la aparición de casos de CE se distribuye de forma uniforme durante el año. La estacionalidad fue reportada en un estudio realizado en España ${ }^{14}$ donde hubo una mayor incidencia de los CE en el verano, alcanzando su máximo en agosto, y la menor en el invierno con mínimo situado en enero. Sin embargo, las razones de este patrón estacional no fueron esclarecidas.

El presente estudio constató que las urgencias por CE son más comunes en los días laborables de la semana. Este hecho contrasta con un estudio efectuado en las urgencias del ORL/HCM donde la mayor parte de los pacientes con CE concurrieron los fines de semana ${ }^{12}$. La mayor ocurrencia de pacientes con CE en los días laborables fue también reportada en un estudio efectuado en España ${ }^{14}$. Las razones de esta aparente disparidad pueden ser varias incluyendo, por ejemplo, un muestreo relativamente más pequeño y criterios de inclusión más específicos que abarcan sólo CE en la faringe y el esófago ${ }^{12}$. Al igual que en la estacionalidad, la literatura es escasa en cuanto a los días de la semana y, por eso, no es posible sacar conclusiones más concretas.

Se verificó que la mayor ocurrencia de CE fue en edades pediátricas, prevaleciendo el sexo mas- 
culino. Estos dos hallazgos confirman lo reportado en la literatura. La literatura internacional reporta un predominio de casos de CE en los niños, sobre todo de causa accidental, debido a los riesgos a que se someten en actividades lúdicas y de entretenimiento en el contexto doméstico, en la comunidad y/0 incluso en las escuelas ${ }^{2,3}$. La literatura sobre la ocurrencia de casos en cuanto al sexo es ambigua, con estudios mostrando no haber diferencia entre ambos sexos ${ }^{3}$ y otros mostrando un claro predominio del sexo femenino ${ }^{14}$. Nuestra experiencia indica que el tipo de CE encontrados en ambos sexos parece tener una relación estrecha con los hábitos, costumbres, prácticas y ocupaciones profesionales. Por ejemplo, las costureras a menudo colocan agujas en la boca y lo mismo sucede con los carpinteros que colocan clavos en la boca durante sus actividades y acaban tragándolos accidentalmente. Los niños también tienen el hábito de colocar objetos en la boca, sobre todo monedas. Este estudio muestra que la mayor parte de los CE eran inorgánicos $(55,34 \%, 2.671 / 4.826)$ mientras que el $44,65 \%(2.155 / 4.826)$ eran orgánicos. Estos resultados se asemejan a un estudio efectuado en la República Dominicana ${ }^{15}$. En el presente estudio, los metales fueron los más predominantes. Un estudio de Sudáfrica confirma nuestros resultados ${ }^{3}$, pero estos hallazgos difieren de otro estudio efectuado en España ${ }^{16}$.

Sin embargo, la literatura señala que el tipo de CE depende, entre otros factores, de los hábitos dietéticos de la población, lo que determina que las espinas sean más frecuentes en la orofaringe y los huesos de aves sean más comunes en el esófago ${ }^{17}$.

Este estudio mostró que entre los CE orgánicos del tipo alimentario, la espina de pescado es el más común. Al ser uno de los principales alimentos de la población, por ser el más accesible que las otras fuentes de proteína alimentaria, parece razonable esperar que partes considerables de la población de este país, particularmente los residentes en la costa, estén expuestas a este riesgo. Comúnmente los peces de tamaño pequeño son relativamente más baratos y son los más consumidos. Sin embargo, estos peces pequeños poseen más espinas y por eso aumentan el riesgo de alojamiento del CE a nivel del tracto digestivo. En un estudio efectuado en Nigeria, las prótesis dentales fueron el segundo CE más frecuente en la vía digestiva, después de las monedas ${ }^{18}$.

En este estudio, el CE del CAE fue la localización anatómica más frecuente. Estos resultados difieren de un estudio de Singapur donde hubo una distribución equitativa de los CE por el CAE, fosas nasales y faringe ${ }^{19}, 0$ de los estudios de la India y de Sudáfrica donde hubo predominio de CE nasal ${ }^{3,9}$.

Observamos que los semillas/granos fueron Ios CE más encontrados en la vía aérea $(27,95 \%$, 45/161). Estos hallazgos son similares a un estudio efectuado en España ${ }^{14}$. Otro estudio realizado en China ${ }^{20}$ mostró que los huesos eran más frecuentes, seguidos de espina y semillas.

En nuestro estudio los CE más encontrados en la vía digestiva, fueron los compuestos de metal $(48,16 \%, 1023 / 2124)$, seguidos de espina de pescado/huesos $(47,45 \%, 1.008 / 2.124)$ y compuestos de plástico $(2,10 \%, 46 / 2.124)$. Estos resultados no concuerdan con un estudio efectuado en España ${ }^{21}$ que encontró más huesos, seguidos de espina de pescado.

A pesar de que nuestro estudio no permite saber en qué lado del CAE/ fosas nasales se encontraba el CE, porque normalmente esta información no viene escrita en los registros, un estudio mostró mayor incidencia en el lado derecho debido al hecho de la mayoría de la población mundial es diestra $^{22}$

En el presente estudio, el CE del esófago fue el segundo más frecuente $(31,90 \%)$ similar a un estudio en España ${ }^{14}(10,30 \%)$. En Ghana, Ios CE esofágicos son la causa más común de emergencia en ORL $(41,30 \%)^{7}$.

Los CE nasales ocupan el tercer lugar en nuestro estudio $(12,3 \%)$ y los faríngeos la cuarta localización más frecuente, mientras que en España ${ }^{4,14}$ son los más predominantes. Las investigaciones apuntan que la mayor parte de los CE faríngeos se encuentra en las amígdalas palatinas y lingual ${ }^{22}$, probablemente debido a la presencia de las criptas que favorecen la retención.

\section{CONCLUSIONES}

Este estudio presenta una recopilación única de casos de pacientes con CE en ORL que abarca un período de 26 años, constituyendo por lo tanto una 
importante fuente de información al respecto. Por otro lado, si tomamos en cuenta las evidencias de este estudio y las evidencias existentes en la literatura, se pueden sacar conclusiones que pueden ser de utilidad para definir estrategias de prevención y control de los incidentes por CE en ONCC. Las diferencias de los tipos de CE involucrados y su magnitud, las regiones anatómicas más afectadas y las variaciones encontradas en cuanto a los grupos de edad y

\section{BIBLIOGRAFIA}

1. Gregori D, Scarinzi C, Berchialla P, et al. The cost of foreign body injuries in the upper aerodigestive tract: Need for a change from a clinical to a public health perspective. Int $J$ Pediatr Otorhinolaryngol 2007; 71: 1391-8.

2. Silva Neto JJ, Lima JC, Vitale RF, et al. Corpos estranhos em Otorrinlaringologia- Levantamento do Hospital Monumento e Clínica Otorhinus. Arq Int Otorrinolaringol 2007; 11: 305-10.

3. van As AB, du Toit N, Wallis L, et Al. The South African experience with ingestion injury in children. International Congress Series 2003; 1254: 287-93.

4. Pardo Mateu L, Martinez P, Garcia J. Cuerpos extraños del área otorrinolaringologica en la infancia. Acta Pediátrica Española 1998; 56(5): 286-90.

5. ISEH K, YAHAYA M. Ear foreign bodies: Observations on the clinical profile in Sokoto, Nigeria. Annals of African Medicine 2008; 7(1): 18.

6. Menchen P, Castellanos D, Senent C, et al. Cuerpos extraños en esófago. Terapéutica endoscópica. Ponencia Oficial. Urgencias en Otorrinolaringología. Acta Otorrinolaring Esp 1991; 42: 69-71.

7. Kitcher ED, Jangu A, Baidoo K. Emergency ear, nose and throat admissions at the Korle-Bu Teaching Hospital. Ghana Medical Journal2007; 41: 9-11.

8. Symvoulakis EK, Klinis S, Alegakis A, et al. Epidemiologic profile of otorhinolaryngological, head and neck disorders in a tertiary hospital unit in Greece: a challenge for general practitioners. sexo reportados en este estudio con relación a la literatura probablemente resultan de los factores contextuales regionales y hospitalarios. Por eso, a pesar de las limitaciones, las evidencias de este estudio pueden ser importantes desde el punto de vista de salud pública en países con características similares a las de Mozambique. La particular importancia se puede revelar por demostrar la magnitud del problema en la edad pediátrica, sobre todo en los niños de 0 a 4 años de edad.

BMC Ear, Nose and Throat Disorders 2006; 6: 12.

9. Kumar S, Gulati A. Pediatric Emergencies in Otolaryngology in a Metropolitan City. Indian Pediatrics 1999; 36: 1256-8.

10. Ibrahimo N.A, Fernandes N. Normas de Atendimento à Criança Sadia e à Criança em Risco. Maputo: GM/MISAU; 2011.

11. Martinez VE. Cuerpos extranos en otorrinopediatria. V Manual de Otorrinolaringologia Pediatrica de la IAPO. Sao Paulo; 2006; 62-4.

12. Dadá MS, Costa JL, Huang H, et al. Urgência de ORL no Hospital Central de Maputo. Revista Portuguesa de Otorrinolaringologia 2004; 38 : 33-40.

13. Tan HKK, Brown K, McGill T, et al. Airway foreign bodies (FB): a 10-year. International Journal of Pediatric Otorhinolaryngology 2000; 56: 91-9.

14. Lopez Amado M, Garcia Sarandeses A, Herranz González-Botas J, et Al. Cuerpos extranos del area $0 . R . L$. Analisis de un ano de asistencia. Anales Otorrinolaringol Ibero Am 1993; 20: 541-53.

15. Ferreiras F, Severino B, Solano L. Frecuencia de Cuerpo Extraño en Nariz, Oídos, Orofaringe. Rev Med Dom 2003; 64: 34-7.

16. García Aa, Guzmán E, Batista FA, Guzmán W. Cuerpos extrano en una Emergencia de Pediatria. Acta Medica Dominicana 1998; 20: 129-33.

17. Benito Navarro J, Cuvillo Bernal A, Porras Alonso E. Cuerpos Extranos esofágicos: nuestra experinecia en diez anos. Acta Otorrinolaring Esp 2003; $54:$ 281-5.

18. BHATIA P.L. Hypopharyngeal and oesophageal foreign bodies. East Afr Med J 1989; 66: 804-11.

19. Ngo A, Ng KC, Sim TP. Otorhinolaryngeal 
foreign bodies in children presenting to the emergency department. Singapore Med J 2005; 46: 172-8.

20. Chen CH, LaI CL, TSAI TT, et al. Foreign bodies aspiration into lower airway in chinese adults. Chest Journal 1997; 112: 129-33.
21. ChIK HH, MIU TY, Chan CW. Foreign body aspiration in Hong Kong Chinese Children. Hong Kong Med J 2009; 15: 6-11.

22. McGuirt WF, Holmes KD, Feehs R, et Al. Tracheobronchial foreign bodies. Laryngoscope 1988; 98: 615-8. 